This is the accepted version of a manuscript that has been uploaded to an institutional repository to meet ARC funding requirements to make this research open access.

The final version of this manuscript can be found at the journal website, Community Mental Health Journal, here: https://link.springer.com/article/10.1007/s10597-017-0217-5

Please cite this article as below:

Johnson SE, Lawrence D, Perales F, Baxter J, \& Zubrick SR. (2017). Prevalence of mental disorders among children and adolescents of parents with self-reported mental health problems. Community Mental Health Journal, 54(6): 884-897. DOI: 10.1007/s10597-017-0217-5 


\section{Prevalence of mental disorders among children and adolescents of parents with self-reported mental health problems}

Sarah E. Johnson ${ }^{\mathrm{a}}$, David Lawrence ${ }^{\mathrm{b}}$, Francisco Perales ${ }^{\mathrm{c}}$, Janeen Baxter ${ }^{\mathrm{c}}$, Stephen R. Zubrick ${ }^{\mathrm{a}, \mathrm{b}}$

aTelethon Kids Institute, The University of Western Australia, 100 Roberts Road, Subiaco, Western Australia, 6008

${ }^{b}$ Graduate School of Education, The University of Western Australia, Crawley, Perth, Western Australia, 6009.

'Institute for Social Science Research, The University of Queensland, Long Pocket Precinct, 80 Meiers Rd, Building C, Indooroopilly, Brisbane QLD 4068.

Corresponding author: Sarah Johnson. Sarah.Johnson@telethonkids.org.au; (+61) 894897715.

Funding: This research was supported by the Australian Research Council Centre of Excellence for Children and Families over the Life Course (project number CE140100027). The views expressed herein are those of the authors and are not necessarily those of the Australian Research Council. This study uses data from Young Minds Matter which was funded by the Australian Government Department of Health.

Acknowledgements: The authors would like to thank the 6,310 families who gave their time to participate in the survey, Rajni Walia, Gerry Bardsley, the team at Roy Morgan Research, and the Survey Reference Group for their input into the design and conduct of the survey. The authors would also like to thank Margaret Cook for her dedication to the issue of improving the lives and opportunities of children whose parents have serious mental health problems, and for her input and guidance in producing this paper. 


\begin{abstract}
This paper provides Australian population-level estimates of the prevalence of parental self-reported lifetime mental disorders and past 12-month mental disorders in their children. It leverages unique data from the 2013-14 Australian Child and Adolescent Survey of Mental Health and Wellbeing (Young Minds Matter) (n=6,310). Mental disorders were assessed in 4-17 year-olds using the Diagnostic Interview Schedule for Children Version IV. Primary carer (PC) and secondary carer (SC) mental health was based on PC-reported lifetime diagnoses. Over one third of 4-17 year-olds had a PC with a lifetime diagnosis. The prevalence of all disorders was significantly higher amongst these children than children whose PC reported no diagnoses, and highest when the PC had comorbid and more severe disorders. Assessing mental health needs at a family level is important to identify children who are particularly vulnerable to developing mental disorders, to develop targeted interventions, and to understand the intergenerational transmission of risk.
\end{abstract}

Keywords: parental mental health; child and adolescent mental health; mental disorders; intergenerational risk; children and families 


\section{Introduction}

Mental disorders are common and burdensome, affecting 10-20\% of young people worldwide (Kieling et al. 2011). During 2013-14, about $14 \%$ of Australian children and adolescents had mental disorders, with rates unchanged from 15 years ago (Lawrence et al. 2015). In 2007, the prevalence of any lifetime mental disorder amongst Australian adults was about $45 \%$ (Slade et al. 2009). The majority of mental disorders have their onset in childhood and adolescence, making early, appropriate and effective intervention critical for the overall improvement of population mental health (Kessler et al. 2007). In this paper we argue that such intervention should not only be focused on the child, but should also consider and address the needs of parents and carers who suffer from mental disorders.

Children of parents with mental disorders are at genetic and environmental risk of developing mental disorders themselves, with their risk being at least twofold compared to children whose parents have no history of mental disorders (Dean and Murray 2010; Hosman et al. 2009; Rasic et al. 2014; Reupert et al. 2012; van Santvoort et al. 2015; Weissman et al. 2006). The risk is associated with both paternal (Ramchandani and Psychogiou 2009) and maternal (Goodman et al. 2010) disorders. Depending on the severity and duration of the problem and access to treatment and support, parents with mental disorders may be less emotionally responsive, more likely to exert poorer parenting practices (such as inconsistent or harsh disciplining), and less likely to monitor their children's activities, or to seek and have access to help (Hosman et al. 2009; Reupert et al. 2012; Ramchandani and Psychogiou 2009; Yoshikawa et al. 2012). The degree of impact of poor parental mental health on the child depends also on other related family risks, such as poverty and social disadvantage, family structure (living in lone parent or reformed families), marital discord, alcohol and drug use, domestic violence, abuse, and family dissolution (Campbell et al. 2012; Perales et al. 2015; Rasic et al. 2014; Yoshikawa et al. 2012). Recent research has also shown that the familial risk for mental health problems can be passed down through multiple generations (Johnston et al. 2013).

Although children share genetic risks for specific mental disorders with their parents, they are also at heightened risk to develop a broad spectrum of other mental disorders (Dean and Murray 2010; Hosman et al. 2009; McLaughlin et al. 2012; Rasic et al. 2014; Reupert et al. 2012; van Santvoort et al. 2015; Weissman et al. 2006). While about one third of offspring of parents with severe mental illness (schizophrenia, bipolar disorder or major depressive disorder) will develop a severe mental illness themselves, more than half will develop any mental disorder (Rasic et al. 2014). On the whole, the risk tends to be greater for developing the same disorder as one's parents, compared to another disorder, although it is difficult to disentangle the relative contribution of 
genetic versus environmental risks (Dean and Murray 2010; Hosman et al. 2009; Rasic et al. 2014; Reupert et al. 2012; van Santvoort et al. 2015; Weissman et al. 2006).

It is currently estimated that about $23 \%$ of Australian children have a parent with a non-substance use 12-month mental disorder (Maybery et al. 2009). While parental substance abuse imparts greater risk of substance abuse and a range of disorders to their offspring (Hosman et al. 2009; Ramchandani and Psychogiou 2009; Straussner and Huff-Fewell 2011), prior research is limited by the lack of information on externalizing disorders in parents. Furthermore, most studies lack information on comorbidity and severity of common disorders, as well as information on more than one parent (Rasic et al. 2014; van Santvoort et al. 2015). Evidence that relies mostly on clinical data shows the prevalence of mental disorders in children to be higher when more than one parent is affected (Dean and Murray 2010; Hosman et al. 2009; McLaughlin et al. 2012).

Population estimates of the number of children exposed to parental mental disorders are rare and mostly sourced from adult population surveys or clinical samples (van Santvoort et al. 2015), limiting comparisons with Australia. Based on prior research, the magnitude and strength of the relationships between specific parental and child diagnoses are also difficult to compare due to different combinations of community and clinical samples, and study methods. Up until now, the requisite data to investigate these issues in the Australian context were not available. Australia provides a useful point of comparison to international studies which have mainly focused on the US and Europe. It shares many of the same economic and demographic characteristics as other western countries such as the UK, US and Western Europe. But unlike some, particularly the US, Australia has relatively strong governmental support for programs and policies to support families, provision of universal health care and relatively high minimum wage rates (Gornick and Jantti 2012; Kalil et al. 2012). Such benefits may mitigate some of the effects of children's exposure to parental mental disorders. However, despite an increase in government spending on mental health services in past decades, there is still a need for major structural reform (National Mental Health Commission 2014). Australia like many countries, faces challenges in implementation, sustainability of funding and State/Territory variation in family-focused policy and practice for children and families of parents with mental illness (Nicholson et al. 2015). Therefore, despite strong governmental support and community advocacy, there is still much to be done to enable early and effective intervention.

In this paper we use recent, nationally representative Australian data from the second Australian Child and Adolescent Survey of Mental Health and Wellbeing (Young Minds Matter) to (i) quantify the proportion of children (ages 4-17) who have parents with lifetime mental health problems, (ii) determine the proportion of 
children whose parents have lifetime mental health problems who have mental disorders themselves, and how this varies by parental diagnoses, (iii) identify if associations with child disorders were stronger for primary or secondary carer diagnoses, and when both carers had problems diagnosed, and (iv) establish how family socioeconomic circumstances vary by parental diagnoses.

\section{Methods}

\section{Population}

Young Minds Matter was conducted during 2013-14 by the Telethon Kids Institute, The University of Western Australia, in partnership with Roy Morgan Research. The study was funded by the Australian Government Department of Health. In total, 6,310 parents and carers across Australia participated in the survey (55\% of eligible households). Where there was more than one child in scope, the study child was randomly selected by computer. Face-to-face interviews were undertaken in households with primary carers ( $84 \%$ female; $81 \%$ biological mothers). The primary carer (PC) was identified as the person in the household who was reported to know the most about the selected child. Of all secondary carers, $82 \%$ were male, and $73 \%$ were biological fathers.

The representativeness of the Young Minds Matter sample was assessed by comparison with demographic data from the 2011 Australian Census of Population and Housing. Such comparison showed that the sample was broadly representative of the Australian population of 4-17 year-olds. The survey data were weighted to represent the full Australian population of 4-17 year-olds, and to adjust for non-response bias. The survey methodology has been described in detail elsewhere (Hafekost et al. 2016).

\section{Variables}

Parental mental health. PC mental health was determined by self-report of ever being diagnosed with a mental health problem by a doctor or mental health professional, using a showcard as a prompt. Where a secondary carer (SC) was present in the household, the PC reported mental health diagnoses on their behalf. Responses were grouped into six hierarchical categories with the aim of addressing comorbidity of common disorders, including the highly comorbid nature of psychotic illness (Campbell et al. 2012; Morgan et al. 2011; Seeman et al. 2015) and alcohol or drug dependence (Straussner and Huff-Fewell 2011) and the greater severity of anxious major depressive disorder compared with non-anxious major depressive disorder in adults (Kessler et al. 2015). The categories were: 
1) 'Bipolar or psychotic disorder' (including schizophrenia, and those with a co-diagnosis in any of the categories below).

2) 'Alcohol or drug dependence' (including those with a co-diagnosis in any of the categories below).

3) 'Anxiety (panic attacks, post-traumatic stress disorder, obsessive-compulsive disorder, and any other anxiety problem) and depression'.

4) 'Depression only'.

5) 'Anxiety disorders only'.

6) 'Other mental health problem' (including ADHD and the remainder with unspecified problems; these may also have a co-diagnosis of depression or anxiety).

PCs were also asked how much mental health problems had interfered with their daily activities (ranging from 'none of the time' to 'all of the time') since the study child was born and asked to report the same, if applicable, for SCs. This question was not conditional on ever being diagnosed with a mental health problem. PCs' level of psychological distress in the four weeks prior to the interview was assessed using the Kessler 10 Psychological Distress Scale (Kessler et al. 2003).

Child and adolescent mental disorders. Twelve-month mental disorders were assessed using the Diagnostic Interview Schedule for Children Version IV (DISC-IV) (Fisher et al. 1993). The DISC-IV implements the criteria for mental disorders set out in the Diagnostic and Statistical Manual of Mental Disorders, $4^{\text {th }}$ edition (American Psychiatric Association, 2000). These criteria are based on sets of clinically significant symptoms associated with impairment of functioning. Seven modules were completed by PCs: anxiety disorders (social phobia, separation anxiety disorder, generalized anxiety disorder, obsessivecompulsive disorder) (6.9\% of children); major depressive disorder (2.8\%), attention-deficit/hyperactivity disorder (ADHD) (7.4\%); and conduct disorder (2.1\%). In all, 13.9\% of 4-17 year-olds had mental disorders. A measure of disorder severity was developed based on questions about the degree to which symptoms were perceived to impact on the child's functioning in four different domains: at school or work, with friends, on family and on self, including personal distress (Zubrick et al. 2015). Of those with mental disorders, 59.8\% of cases were mild, $25.4 \%$ moderate, and $14.7 \%$ were severe (Lawrence et al. 2015).

Socioeconomic factors. A series of measures was used to describe socioeconomic circumstances of families in which parents had mental health problems. Annual gross household income (from all sources including wages, investments and government pensions), highest level of parent or carer education and labour force status were collected using the Australian Bureau of Statistics (ABS) standard formats. A dichotomous 
variable capturing whether families lived below or above a poverty line was derived from the household income question. Gross household income was estimated as the mean income of the category obtained from fitting a normal distribution to the income data. A measure of equivalised income was then calculated using OECD standards (Australian Council of Social Services 2014). Finally, a poverty line was estimated at $50 \%$ or below of the median gross equivalised household income $($ YMM median $=\$ 39,534)$. Using this measure, $21.3 \%$ of children lived below the poverty line.

Area-level socio-economic (dis)advantage was taken from the 2011 ABS Index of Relative SocioEconomic Disadvantage (IRSED) for the Statistical Area 1 (SA1) in which the family resided (Australian Bureau of Statistics, 2013).

Sole-parent status $(20.3 \%)$ was determined by a family relationships grid. We also determined if the household had no parent employed - either the sole parent, or both in a two-parent family (12.0\%). For the purposes of this analysis, a dichotomous variable was created indicating if the young person lived in the lowest (most disadvantaged) quintile or not (17.7\%). The survey also included a set of four questions about shortage of money in the past 12 months and responses were categorized into having two or more indicators of financial strain $(10.8 \%)$ or not.

\section{Analysis}

All proportions have been weighted to represent 4-17 year-olds in the Australian population. Where compared, proportions across groups were determined to be significantly different when $95 \%$ confidence intervals did not overlap, or if they did overlap, were found to be significantly different by calculating confidence intervals for the difference between estimates. Differences between proportions of children with mental disorders by PC diagnosis relative to PCs with no diagnosis were determined by calculating relative risks with $95 \%$ confidence intervals by specifying $2 \times 2$ tables.

\section{Compliance with Ethical Standards}

Ethical approval. The research protocol for Young Minds Matter was approved by two institutional review boards - the Australian Government Department of Health Human Research Ethics Committee (Project 17/2012), and The University of Western Australia Human Research Ethics Committee (Project RA/4/1/5538). All research has been undertaken in accordance with Australia's National Statement on Ethical Conduct in Human Research.

Conflict of interest statement. The authors have no conflict of interest to report and certify responsibility for the manuscript. 


\section{Results}

\section{Prevalence of PC and SC Mental Health Problems}

More than one third of PCs had ever been diagnosed with a mental health problem (37.2\%), most commonly with anxiety disorders $(8.2 \%)$, depression $(13.1 \%)$ or both anxiety and depression $(10.6 \%)$, and less frequently with alcohol or drug dependence $(1.6 \%)$, a bipolar or psychotic disorder $(1.5 \%)$, or another unspecified problem (2.3\%) (Table 1). The large majority of PCs with a bipolar or psychotic disorder (88.6\%) or alcohol or drug dependence $(84.5 \%)$ also had other mental health diagnoses - the most common comorbid conditions were anxiety and depression.

One fifth of PCs ever diagnosed with a mental health problem $(22.7 \%)$ had high or very high levels of psychological distress (in the last 4 weeks) and 10\% reported that mental health problems had interfered with activities 'most or all of the time' since the child's birth. Amongst those PCs with a bipolar or psychotic disorder, the analogous figures were $44 \%$ and $30 \%$.

A higher proportion of PCs in single-parent families compared to those in two-parent families had ever been diagnosed with a mental health problem (50.2\% compared to $34.1 \%)$, with rates significantly higher across most categories. High to very high distress levels were also more prevalent amongst PCs in single-parent families with a mental health diagnosis (31.2\% compared to $19.7 \%$ of PCs with mental health problems in twoparent families).

Two-parent families. Twenty percent of SCs had ever been diagnosed with a mental health problem, most commonly with anxiety disorders $(4.2 \%)$, depression $(8.6 \%)$ or both anxiety and depression $(3.0 \%)$, and smaller proportions with alcohol or drug dependence (1.8\%) a bipolar or psychotic disorder (1.0\%) or another unspecified mental health problem (1.4\%) (Table 1). Altogether, 43.1\% of 4-17 year-olds in two-parent families had at least one parent or carer who had ever been diagnosed with a mental health problem. Additionally, in $11.0 \%$ of two-parent families, both parents or carers had ever been diagnosed with a mental health problem (not shown in the tables).

\section{Family Socioeconomic Circumstances}

Relative to children living with PCs with no history of mental health problems, children living with PCs who had ever been diagnosed with a mental health problem were more likely to be living below the poverty line, in disadvantaged geographical areas, jobless households, and sole-parent families, and to have experienced financial strain over the past 12 months (Table 2). Children of PCs ever diagnosed with a bipolar or psychotic 
disorder or alcohol or drug dependence were significantly more likely to live in disadvantaged circumstances compared to children of PCs with any diagnosis.

\section{Prevalence of Any Child Mental Disorder by PC and SC Mental Health Diagnoses}

Child mental disorders were more prevalent when the PC had ever been diagnosed with a mental health problem compared to a PC with no history of diagnosis $(22.6 \% \text { compared to } 8.8 \% \text {; RR=2.6, 95\% CIs 2.2-3.0) (Table } 3)^{1}$

The prevalence of any child disorder was about fourfold when the PC had been diagnosed with a bipolar or psychotic disorder, alcohol or drug dependence, or another (unspecified) mental health problem, compared to a PC with no history of diagnosis.

There was a higher prevalence of child mental disorders when the PC had ever been diagnosed with a mental health problem in both sole-parent (30.0\% compared to $14.6 \%)$ and two-parent families $(19.1 \%$ compared to $7.3 \%$ ). There were also differences by family type with respect to the specific PC diagnoses. If the PC had a bipolar or psychotic disorder, the prevalence of child and adolescent diagnoses was twice as high in sole-parent than two-parent families $(51.6 \%$ compared to $24.0 \%$; $\mathrm{p}=0.012)$. If the PC was ever diagnosed with anxiety only, depression only or the combination of both disorders, rates of child mental disorders were about $50 \%$ higher in the sole-parent than two-parent families $(\mathrm{p}=0.067, \mathrm{p}=0.013$, and $\mathrm{p}=0.014$ respectively).

Two-parent families. Where only the SC was diagnosed with any mental health problem, the prevalence of child mental disorders was significantly lower relative to when just the PC was diagnosed $(10.6 \%$ compared to $19.1 \%$ ). Relative to where neither the PC nor the SC had a mental health diagnosis, there was a 2.6 times greater prevalence $(95 \% \mathrm{CI}=2.2-3.2)$ of child mental disorders when only the PC was diagnosed, a 1.5 times greater prevalence $(95 \% \mathrm{CI}=1.1-2.0)$ when only the $\mathrm{SC}$ was diagnosed, and 3.0 times greater prevalence when both carers were diagnosed $(95 \% \mathrm{CI}=2.4-3.8)$. When both the PC and the SC had mental health diagnoses, the rate of child mental disorders was not significantly higher compared to when the PC but not the SC was diagnosed $21.9 \%$ compared to $19.1 \%, \mathrm{p}=0.240$ ) but was significantly higher when only the SC was diagnosed $(21.9 \%$ compared to $10.6 \%, \mathrm{p}=0.000)$.

\section{Prevalence of Specific Child Mental Disorders by PC and SC Mental Health Diagnoses}

Overall, the prevalence of child anxiety disorders was about 3.5 times higher when the PC had any lifetime diagnosis, in contrast to when the PC had no diagnoses (12.5\% v 3.6\%) (Table 4). Compared to families in

\footnotetext{
${ }^{1}$ Additional analysis identifying the gender of the PC showed that the prevalence of mental disorders in 4-17 year-olds was approximately twofold when the PC was the (biological, adoptive, step or foster) mother $(n=5,193)$ compared to if the PC was the father ( $n=1,013)$. A small number of children had PCs who were grandparents or other family members $(\mathrm{n}=104)$. If the PC was the mother, the prevalence of child mental disorders was $23.5 \%(95 \% \mathrm{CIs}=21.3-25.7 \%)$ and $9.5 \%(95 \% \mathrm{CIs}=8.4-10.7 \%)$ respectively for PCs with any mental health diagnosis and no diagnosis respectively $(\mathrm{RR}=2.5)$. The comparable prevalence figures if the PC was the father were $11.3 \%(95 \% \mathrm{CIs}=7.0$ $15.6 \%)$ and $4.8 \%(95 \% \mathrm{CIs}=3.0-6.5 \%)$ respectively $(\mathrm{RR}=2.4)$. These differences were observed in both single and two-parent families.
} 
which PCs had no diagnosis, in families where the PC had an anxiety disorder only, depression only, or both anxiety and depression, rates of child anxiety were approximately twofold (8.4\%), threefold (9.8\%) and fourfold (15.4\%), respectively. The prevalence of child anxiety disorders were about sevenfold (26.1\%) when the PC had a diagnosis of bipolar or psychotic disorder; more than fourfold with a diagnosis of alcohol or drug dependence (15.1\%), and about fivefold with another unspecified mental health problem (19.1\%).

The prevalence of child major depressive disorder was 3.4 times higher when the PC had any lifetime diagnosis of a mental health problem than when the PC had no diagnoses (5.0\% compared to $1.5 \%)$. In all families in which the PC had an anxiety disorder only, depression only, or anxiety and depression, rates of child major depressive disorder were approximately twofold (3.2\%), threefold (4.4\%) and fourfold (5.9\%), respectively, compared to families in which PCs had no diagnosis. The prevalence of child major depressive disorder was threefold when the PC had a diagnosis of bipolar or psychotic disorder (4.5\%), eightfold with a diagnosis of alcohol or drug dependence (12.0\%), and 4.5 times higher with another mental health problem (6.6\%).

The prevalence of child ADHD or conduct disorder was 2.5 times higher when the PC had any lifetime diagnosis of a mental health problem in contrast to when the PC had no such diagnosis. Rates of child ADHD or conduct disorders were about twofold if their PCs had been diagnosed with either anxiety or depression alone $(9.7 \% ; 10.4 \%)$, and threefold if the PC was ever diagnosed with both anxiety and depression $(14.5 \%)$. The prevalence of ADHD or conduct disorders in children were about fourfold when the PC had a diagnosis of bipolar or psychotic disorder $(22.8 \%)$ or of alcohol or drug dependence $(22.6 \%)$. The prevalence of child ADHD or conduct disorder when the PC had a diagnosis in the 'other mental health problem' category was $29.6 \%$; twice as high as if the PC had any mental health problem (13.6\%), and more than fivefold if the PC had no diagnosis $(5.4 \%)$.

Two-parent families. The prevalence of child anxiety disorders, major depressive disorder and ADHD or conduct disorders were all significantly lower at $\mathrm{p}<0.05$ when only the SC had any lifetime diagnosis than when just the PC had been diagnosed. The highest rates of child anxiety disorders by SC diagnosis were associated with alcohol or drug dependence (19.1\%) whilst the highest rates of ADHD or conduct disorders were associated with the SC being diagnosed with another mental health problem (23.4\%). If both the PC and the SC had been diagnosed with any mental health problem, the prevalence of all disorders was not significantly higher than if only the PC had been diagnosed. 


\section{Severity of Child Mental Disorder by Severity of PC Mental Health Problem}

The prevalence of any child disorder increased with the functional impact of the PCs' mental health problem, rising as high as $46.1 \%$ when the PC had ever been diagnosed with a mental health problem and which interfered with her/his daily activities most or all of the time (Table 5). Even without a professional diagnosis, if the PC reported at least some interference from mental health problems, one third of children in these families $(32.5 \%)$ had a mental disorder. Only a small proportion of children whose PCs had been diagnosed with a mental health problem which had little to no interference with functioning had a severe disorder (1.3\%), less than if problems interfered some $(5.5 \%)$ or most or all of the time $(13.1 \%)$.

\section{Discussion}

In this paper we have provided a comprehensive and unique overview of the associations between parental and child mental health problems in an Australian nationally representative sample of 4-17 year-olds. Australia provides an important point of comparison to other western countries where most research on this issue has focused. We show that it is relatively common for children and adolescents to have a parent with a history of mental health problems, with over a third of 4-17 year-olds having a primary carer ever diagnosed and more than two-fifths having either a primary or secondary carer with a mental health diagnosis in two-parent families. This figure is consistent with earlier estimates of $23 \%$ of children having a parent with a mental disorder in the preceding 12-month period (Maybery et al. 2009), as lifetime prevalence of mental disorder in adults is more than double that of 12-month prevalence (Slade et al. 2009). Our results also demonstrate greater risks of mental disorders for children when parents have more severe and comorbid disorders, and where parents report that these disorders interfere with their daily functioning. These conditions are concomitantly associated with greater socioeconomic adversity, which may further contribute to the risk for childhood disorders.

Consistent with previous studies, the prevalence of any child mental disorder doubled when the primary carer had ever been diagnosed with a mental health problem (Dean and Murray 2010; Hosman et al. 2009; Rasic et al. 2014; Reupert et al. 2012; van Santvoort et al. 2015; Goodman et al. 2010). In line with previous reviews reporting children to be at risk for a broad spectrum of disorders, we show that all primary carer diagnoses were associated with significantly higher rates of anxiety disorders, major depressive disorders and ADHD or conduct disorders in 4-17 year-olds (Dean and Murray 2010; McLaughlin et al. 2012; Rasic et al. 2014; van Santvoort et al. 2015). Where there was information on the same disorders for parents and children, that is, anxiety and depression, we did not find a greater risk for children having a diagnosis in the same group. 
We did, however, identify that child internalizing disorders (anxiety disorders and major depressive disorders) were more strongly related to parental depression than child externalizing problems (ADHD and conduct disorders), especially with a comorbid anxiety diagnosis. Likewise, in the WHO World Mental Health Surveys, parental depression, generalized anxiety disorder and panic disorder had a similar strength of association with offspring mood and anxiety disorders (McLaughlin et al. 2012). Furthermore, child externalising disorders were highest when either parent had another mental health problem that included ADHD, although numbers were too few to pinpoint the specific relationship between parent and child ADHD. Others have reported a tendency for the association between the same parental and child disorders to be strongest, especially for more severe disorders (Dean and Murray 2010; Hosman et al. 2009; McLaughlin et al. 2012; Rasic et al. 2014; Reupert et al. 2012; van Santvoort et al. 2015; Weissman et al. 2006). Disorder-specific comparisons were limited in the Young Minds Matter survey due to the use of different measures of mental health problems in parents (selfreported lifetime diagnosis) and children (12-month diagnostic instrument), and because of the younger age of onset of ADHD and anxiety disorders compared to depression. These findings prompt the need for early screening for a broad set of internalising and externalising problems among children who have a parent with a diagnosed mental disorder, sensitive to the average age of onset of specific childhood disorders. For example, it may be unlikely that depressive symptoms are picked up in children before early adolescence, but knowledge that a parent has been diagnosed with major depressive disorder, especially if comorbid with anxiety or other disorders, should trigger screenings for symptoms of anxiety, or attention or hyperactivity problems that tend to onset in childhood. In Young Minds Matter, anxiety disorders were equally prevalent among children and adolescents (about 7\%), whilst ADHD was more prevalent in children than adolescents $(8.2 \%$ and $6.3 \%$ respectively) (Lawrence et al. 2016). Early detection and intervention are key mechanisms for helping to reduce the severity and persistence of problems into adulthood, potentially impacting on all aspects of life.

We addressed a limitation of previous studies by accounting for some of the comorbidity in parental diagnoses. In doing so, we found that the lowest rates of child anxiety disorders were evident when the primary carer had been diagnosed with an anxiety disorder without another comorbid mental disorder, whereas the highest rates were associated with bipolar or psychotic disorders. There may have been further differences in rates of child and adolescent anxiety according to whether parents had a bipolar disorder, or schizophrenia or other psychotic disorder, as others have shown (Rasic et al. 2014). However, we lacked statistical power to test this distinction in a robust way. Furthermore, having a parent (i) with comorbid anxiety and depressive disorders, (ii) for whom impact on functioning was greater, and (iii) for whom current distress levels were 
higher, was associated with a higher prevalence of anxiety in children and adolescents than if the parental diagnosis was anxiety alone. These findings illustrate the importance of considering comorbidity and severity of parental disorders, as identified by others (Hosman et al. 2009; Reupert et al. 2012; Ramchandani and Psychogiou 2009).

The greatest risk for child disorders by parental diagnosis was associated with more severe parental mental disorders, with one third of children having a mental disorder when their primary carer had either a bipolar or psychotic disorder, or alcohol or drug dependence. By comparison, in their meta-analysis of parents with severe mental illness, Rasic and colleagues estimated that $57 \%$ of children age $10-19$ years had any mental disorder, with little variation by parental diagnoses (Rasic et al. 2014). The relatively lower (although still substantial) prevalence of child disorders in our study when primary carers had a severe mental health problem may be due to our estimate being based on a population community sample, inclusion of younger and nonbiological children, and restriction to 12-month disorders. In the Australian Survey of High Impact Psychosis, half of fathers and one third of mothers with psychotic illnesses had a level of impaired independent functioning that was rated as a moderate to severe disability (Campbell et al. 2012). Furthermore, low prevalence disorders are highly comorbid with other mental health problems, including depression and anxiety, and substance abuse (Campbell et al. 2012; Morgan et al. 2010; Seeman et al. 2015). Children in these families are more likely to experience multiple disadvantages. They are thus not only challenged by their parents' mental health problems and their consequences in terms of family stress and more inconsistent parenting (Grant et al. 2006), but also by being poor, lacking essentials and being at greater risk of experiencing stigma and social exclusion (Yoshikawa et al. 2012; Brooks-Gunn and Duncan 1997; Elliott 2016; Pascoe et al. 2016; Ridge 2011). For those parents with psychotic illness, the parenting role may be additionally challenged by the sedative effects of antipsychotic treatment and the need for hospitalisation due to multiple episodes (Seeman et al. 2015). Therefore, reducing the risk for children in these families requires not only providing medical assessment, but also ensuring that further institutional support is provided to these families (e.g. parenting support and other social services).

Family context also mattered. For instance, being in a sole-parent family was associated with a greater prevalence of child disorders regardless of parental diagnosis, particularly when the sole parent had a mental health diagnosis. There were also other differences by family structure and parental diagnoses. Although only a small proportion of all primary carers had a bipolar or psychotic disorder (1.5\%), more than two-fifths were sole parents, and the prevalence of child disorders was twice the size in sole-parent families compared to two-parent families. This may be due to a number of reasons, including the experience of separation and divorce, time 
stress, unemployment and welfare reliance amongst those with a psychotic illness, along with housing instability and risk of social isolation (Campbell et al. 2012; Seeman et al. 2015). Sole parents with severe mental disorders may lack support from the other biological parent, or the presence of a partner to seek medical and other assistance, and provide care when the parent is hospitalised (Reupert et al. 2012; Seeman et al. 2015). Furthermore, older children in sole-parent families may be more likely to be left in the role of caring for their mentally unwell parent, with negative impacts on their own health, academic and social functioning (Reupert et al. 2012). Although all children are at heightened risk for mental disorders when a parent has been diagnosed, particular attention should be given to assessing the mental health and needs of children in sole-parent families when that parent has a mental disorder.

In two-parent families, we further sought to determine whether associations with child mental disorders differed according to whether it was the primary or secondary carer who had a lifetime diagnosis, and whether children were at greater risk if both carers in two-parent families were affected (about 11\% of all 4-17 yearolds). First, we found that child mental disorders were more strongly associated with a mental health diagnosis in primary carers compared to secondary carers. There are few studies that include two parents or carers. McLaughlin et al. (2012) reported mostly no difference in the risk for disorders in offspring according to whether it was the mother or father who had mental health problems. In their review Ramchandani and Psychogiou (2009) also report a similar magnitude of risk associated with maternal and paternal psychiatric disorders overall, although differences were evident depending on the parental and child disorder. For example, evidence points to a stronger association between maternal psychopathology and internalizing problems in children compared to paternal psychopathology (but not for externalising problems in children); the magnitude of the difference is however small and varies by a number of moderating factors, including child age (Connell \& Goodman 2002; Ramchandani and Psychogiou 2009). Indeed, when we identified the gender of the primary carer, the risk of child disorders associated with parental lifetime history of mental health problems compared to no problems was similar if the parent was the mother or father. Second, whilst having two parents versus one parent with a psychiatric diagnosis tends to confer a greater risk for disorders in offspring (Dean and Murray 2010; Hosman et al. 2009; McLaughlin et al. 2012), we found this to be the case only when contrasting both parents versus the secondary carer only. As our focus is on two-parent families, our comparisons between parents are influenced by the inclusion of non-biological parents, and limited by the lack of information about non-resident parents who may have a disorder. It is possible that if only the secondary carer is experiencing mental health problems, that the primary carer is able to some extent, buffer the child against negative 
environmental effects. It could be the case that in families where one carer is seriously impaired, that the other carer has taken on the primary caring role. Whereas, if only the primary carer is affected, and the child spends more time with that parent, perhaps due to the other parent working full-time, the child may be more vulnerable to potentially negative effects (such as poorer parenting and reduced emotional responsiveness). Apart from mental health treatment, targeted family interventions can assist to address the needs of those parents with primary caring responsibility, and equip other family members to support them in that role in the context of mental health problems.

\section{Strengths and Limitations}

This study has a number of strengths. It is based on a nationally representative sample of Australian children and includes an internationally recognised diagnostic measure of mental disorders in children and adolescents. It also includes a number of measures of mental health of both the primary and secondary carer, and diagnoses are grouped with attention to severity and comorbidity, distinguishing between low prevalence disorders and common disorders with and without co-morbidities. In this population sample, we are further able to compare rates of child and adolescent disorders in those whose parents have disorders versus parents with no reported history of mental disorders, contrasts often missing in clinical and high-risk samples.

One limitation is the reporting of mental health problems in secondary carers and the child by the primary carer, which could lead to measurement error or introduce bias to the estimates. It is possible that parents with mental disorders are more likely to be cognizant of the symptoms in their children and therefore better able to accurately identify mental health problems. Conversely, the opposite is also possible, where the parent is more likely to over-report their own symptoms in their children (Ordway 2011). Maternal self-report of depressive symptoms is associated with over-reporting of externalizing and internalizing problems in their children which in turn, overestimates the correlation between maternal depression and mental health problems in children (Fergusson et al. 1993; Najman et al. 2001; Ordway 2011). However, the magnitude of this overestimation bias is difficult to assess without a "gold standard" measure of disorder in children or agreement about how to weight discrepant reports from different informants. An Australian community study assessing the effect of maternal and paternal distress on reporting of behavioural problems in children (using a latent variable based on reports from three informants as the gold standard) found the magnitude of the bias to be very small (Sawyer et al. 1998). Bias may increase with the level of mental health impairment in the parent (Najman et al. 2001). In additional analysis, we identified that the prevalence of any mental disorder was approximately twofold if the primary carer was the mother compared to the father. This was observed regardless of whether the 
primary carer had a mental health diagnosis, thus indicating a gender bias in reporting on the one hand, but on the other hand, a similar heightened risk for childhood mental disorders associated with parental mental health diagnoses for primary carer mothers and fathers. Importantly, the gender bias does not necessarily indicate overreporting of emotional or behavioural problems by the mother, rather a different perspective.

Similarly, parents with mental health problems may over-report problems in their partner.

Alternatively, regardless of whether the primary carer had a mental disorder, problems in secondary carers may be underreported if the primary carer was unaware of problems. There was no means in the survey of checking the validity of the self-reported diagnoses and there are too few studies on which to assess the magnitude of differences in self- versus partner-report of mental health diagnoses (Connell \& Goodman, 2002). To evaluate the magnitude and direction of the possible bias introduced by primary carers reporting mental health problems on behalf of secondary carers, future surveys of mental health could consider interviewing a subset of secondary carers on their own mental health, and on that of their partner. To specifically evaluate the effects of both biological parents, the self-reported mental health of non-resident parents would also need to be included wherever possible. Requests to link with Medicare and State-level health administrative data for both parents in the household could also be considered as a means of attaining objective measurements of primary and secondary carer mental health problems, although these would be limited to health service contacts. Future research in this area is needed. Nevertheless it is rare for population surveys of this kind to interview all household members and we are confident that our data provide unique and important insight into the associations between parental and child mental disorders in Australia.

\section{Implications for Policy and Practice}

We find evidence of strong, population-level links between the mental health of parents and their children in the contemporary Australian context. This adds weight to an already sizeable literature highlighting the need to actively identify and support both the parents and their children in the presence of mental illness. Results from the 2007 National Survey of Mental Health and Wellbeing identified that just a third of adults with 12-month disorders had used health services in the past 12 months $-40.7 \%$ of females and $27.5 \%$ of males (Slade et al. 2009). This suggests that well over half of parents with a mental disorder may have an untreated mental illness. If parents have a history of mental health problems they could be more or less inclined to seek help for their child depending on their help-seeking behaviours and the severity of their own problems (Logan and King 2001). Parental mental illness, such as depression, is both a prevalent and a modifiable risk factor with the potential to reduce risks and developmental problems for children (Weissman 2016). 
Mental health treatment for parents, parenting support and family interventions can reduce the risk of intergenerational impacts on children (Hosman et al. 2009; The Royal Australian and New Zealand College of Psychiatrists 2016; Reupert et al. 2012). It may be difficult to recognise and assess children's needs when parents are attending adult mental health services (Stallard et al. 2004). Meta-analyses of preventive interventions in mentally ill parents (mostly from trials in the US among mothers with affective disorders) show that they may be effective in reducing internalizing and to a lesser extent, externalizing symptoms in offspring (Siegenthalar et al. 2012). Interventions that have proven successful in reducing parental and child symptoms include those that are specific to the condition and/or severity of symptoms; target parenting (specifically, parenting in the context of mental health issues), communication skills and coping skills; and address poverty and other contextual factors by connecting parents with support services in key areas such as child care, housing and employment (Suarez et al. 2016; Yoshikawa et al. 2012). Different models of family-oriented psychoeducation among relatively healthy children of parents with mood disorders have also proven to be successful in improving family functioning and a reduction in child internalizing symptoms (Beardslee et al. 2003; 2007). More specialised programs are available for children at risk from abuse and neglect due to their parents suffering more serious mental health or drug and alcohol problems (Coates and Howe 2016). Given finite resources in mental health, school and other services, the need for screening is most urgent amongst children whose parents have more severe or comorbid disorders, if it is the primary carer of the child who is affected, if the child lives in a sole-parent family in which the parent has a mental illness, or if the child lives in challenging or disadvantaged circumstances. Conversely, it is recommended that parents are screened for prior diagnoses and current symptoms when their child presents for mental health problems, as a means to identify any unmet needs for treatment and support (The Royal Australian and New Zealand College of Psychiatrists 2016; Reupert et al. 2012). 


\section{References}

American Psychiatric Association. (2000). Diagnostic and Statistical Manual of Mental Disorders, Fourth Edition, Text Revision. Washington, DC: American Psychiatric Association.

Australian Bureau of Statistics. (2013). Census of Population and Housing: Socio-economic indicators for areas (SEIFA), Australia, 2011. Canberra: Australian Bureau of Statistics, Cat. 2033.0.55.001.

Australian Council of Social Services. Poverty in Australia, 2014. Strawberry Hills, NSW:ACOSS.

Beardslee, W.R., Gladstone, T. R. G., Wright, E. J. (2003). A family-based approach to the prevention of depressive symptoms in children at risk: evidence of parental and child change. Pediatrics, 112, E99-111.

Beardslee, W. R., Wright, E. J., Gladstone, T. R. G., \& Forbes, P. (2007). Long-term effects from a randomized trial of two public health preventive interventions for parental depression. Journal of Family Psychology, 21(4), 703-713.

Brooks-Gunn, J., \& Duncan, G. J. (1997). The effects of poverty on children. The Future of Children. Children and Poverty, 7(2), 55-71.

Campbell, L., Hanlon, M-C., Poon, A. W. P., Paolini, S., Stone, M., Galletly, C... Cohen, M. (2012). The experiences of Australian parents with psychosis: The second Australian national survey of psychosis. Australian and New Zealand Journal of Psychiatry, 46(9), 890-900.

Coates, D., \& Howe, D. (2016). An evaluation of a service to keep children safe in families with mental health and/or substance issues. Australasian Psychiatry, 24, 483-488.

Connell, A. M., \& Goodman, S. H. (2002). The association between psychopathology in fathers versus mothers and children's internalizing and externalising behaviour problems: a meta-analysis. Psychological Bulletin, 128(5), 746-773.

Dean. K., \& Murray, R. M. (2010). Full spectrum of psychiatric outcomes among offspring with parental history of mental disorder. Archives of General Psychiatry, 67, 822-829.

Elliott, I. (June 2016). Poverty and mental health: A review to inform the Joseph Rowntree Foundation's AntiPoverty Strategy. London: Mental Health Foundation.

Fergusson, D. M., Lynskey, M. T., \& Horwood, L. J. (1993). The effect of maternal depression on maternal ratings of child behaviour. Journal of Abnormal Child Psychology, 21(3), 245-269.

Fisher, P.W., Shaffer, D., Paicentini, J. C., Lapkin, J., Kafantaris, V., Leonard, H., \& Herzog, D. B. (1993). Sensitivity of the Diagnostic Interview Schedule for Children, 2nd edition (DISC-2.1) for specific diagnoses of children and adolescents. Journal of the American Academy of Child and Adolescent Psychiatry, 32, 666-673.

Goodman, S, H., Rouse, M. H., Connell, A. M., Robbins Broth, M., Hall, C. M., \& Heyward, D. (2010). Maternal depression and child psychopathology: A meta-analytic review. Clinical Child and Family Psychology Review, 14, 1-27.

Gornick, J. C., \& Jantti, M. (2012). Child poverty in cross-national perspective: Lessons from the Luxembourg Income Study. Children and Youth Services Review, 34, 558-568.

Grant, K, A., Compass, B. E., Thurm, A. E., McMahon, S. D., Gipson, P.Y., Campbell, A. J...Westerholm, R. I. (2006). Stressors and child and adolescent psychopathology: evidence of moderating and mediating effects. Clinical Psychology Review, 26, 257-283. 
Hafekost, J., Lawrence, D., Boterhoven de Haan, K., Johnson, S. E., Saw, S., Buckingham, W. J... Zubrick, S. R. (2016). Updating the methodology of the Child and Adolescent Component of the Australian National Survey of Mental Health and Wellbeing. Australian and New Zealand Journal of Psychiatry, 50, 866-875.

Hosman, C. M. H., van Doesum, K. T. M., \& van Santvoort, F. (2009). Prevention of emotional problems and psychiatric risks in children of parents with a mental illness in the Netherlands: 1 . The scientific basis to a comprehensive approach. Australian e-Journal for the Advancement of MH, 8(3), 250-263.

Johnston, D. W., Schurer, S., \& Shields, M.A. (2013). Exploring the intergenerational persistence of mental health: Evidence from three generations. Journal of Health Economics, 32,1077-1089.

Kalil, A., Haskins, R., \& Chesters, J. 2012. Investing in Children.:Work, education, and social policy in two rich countries. Brookings Institution Press, Washington D.C.

Kessler, R. C., Amminger, P., Aguilar-Gaxiola, S., Alonso, J., Lee, S., \& Ustun, T. B. (2007). Age of onset of mental disorders: A review of recent literature. Current Opinion in Psychiatry, 20(4), 359-364.

Kessler, R. C., Barker, P. R., Colpe, L. J., Epstein, J. F., Gfroerer, J. C., Hiripi, M. J... Zaslavsky, A. M. (2003). Screening for serious mental illness in the general population. Archives of General Psychiatry, 60, 184189.

Kessler, R. C., Sampson, N. A., Berglund, P., Gruber, M. J., Al-Hamzawi, A., Andrade, L... Wilcox, M.A. (2015). Anxious and non-anxious major depressive disorder in the World Health Organization World Mental Health Surveys. Epidemiology and Psychiatric Sciences, 24(3), 210-226.

Kieling, C., Baker-Henningham, H., Belfer, M., Conti, G., Ertem, I., Omigbodun, O... Rahman, A. (2011). Child and adolescent mental health. Lancet, 378, 1515-1525.

Lawrence, D., Johnson, S., Hafekost, J., Boterhoven de Haan, K., Sawyer, M., Ainley, J., \& Zubrick, S. R. (2015). The Mental Health of Children and Adolescents: Report on the Second Australian Child and Adolescent Survey of Mental Health and Wellbeing. Department of Health: Canberra.

Logan, D. E., \& King, C. A. (2001). Parental facilitation of adolescent mental health service utilisation: A conceptual and empirical review. Clinical Psychology Science and Practice, 8(3), 319-333.

Maybery, D. J., Reupert, A. E., Kent, P., Goodyear, M., \& Crase, L. (2009). Prevalence of parental mental illness in Australian families. Psychiatric Bulletin, 33(1), 22-26.

McLaughlin K. A., Gadermann, A. M., Hwang, I., Sampson, N. A., Al-Hamzawi, A., Andrade, L. H...Kessler, R. C. (2012). Parent psychopathology and offspring mental disorders: results from the WHO World Mental Health Surveys. The British Journal of Psychiatry, 200, 290-299.

Morgan, V., Waterreus, A., Jablensky, A., Mackinnon, A., McGrath, J. J., Carr, V.... Saw, S. People living with psychotic illness 2010. Report on the second Australian national survey. November 2011. Commonwealth of Australia: Canberra.

Najman, J. M., Williams, G. M., Nikles, J., Spence, S., Bor, W., O’Callaghan, ... Shuttlewood, G. J. (2001). Bias influencing maternal reports of child behaviour and emotional state. Social Psychiatry and Psychiatric Epidemiology, 36(4), 186-94.

National Mental Health Commission. (2014). The National Review of Mental Health Programmes and Services. Sydney: NMHC.

Nicholson, J., Reupert, A., Grant, A., Lees, R., Maybery, D., Mordoch, E... Diggins, M. (2015). The policy context and change for families living with parental mental illness. In Reupert, A., Maybery, D., 
Nicholson, J., Gopfert, M., Seeman, M. V. (Eds), Parental psychiatric disorder: distressed parents and their families, (3rd Ed.) (pp. 354-364). Cambridge: Cambridge University Press.

Ordway, M. R. (2011). Depressed mothers as informants on child behaviour: methodological issues. Research in Nursing and Health, 34(6), 520-532.

Pascoe, J. M., Wood, D. L., Duffee, J. H., \& Kuo, A. (2016). Committee on Psychosocial Aspects of Child and Family Health, Council on Community Pediatrics. Mediators and adverse effects of child poverty in the United States. Pediatrics, 137(4), e20160340.

Perales, F., Johnson, S. E., Baxter, J., Lawrence, D., \& Zubrick, S. R. (2016). Family structure and childhood mental disorders: new findings from Australia. Social Psychiatry and Psychiatric Epidemiology, 52, 423433.

Ramchandani, P., \& Psychogiou, L. (2009). Paternal psychiatric disorders and children's psychosocial development. Lancet, 374, 646-53.

Rasic, D., Hajek, T., Alda, M., \& Uher, R. (2014). Risk of mental illness in offspring of parents with schizophrenia, bipolar disorder, and major depressive disorder: a meta-analysis of family high-risk studies. Schizophrenia Bulletin, 40(1), 28-38.

Reupert, A. E., Cuff, R., Drost, L., Foster, K., van Doesum, K. T. M., \& van Santvoort, F. (2012). Intervention programs for children whose parents have a mental illness: a review. Medical Journal of Australia, Open 1 Supp1, 18-23.

Reupert, A. E., Maybery, D. J., \& Kowalenko, N. M. (2012). Children whose parents have a mental illness: prevalence, need and treatment. Medical Journal of Australia, Open1 Suppl, 7-9.

Ridge, T. (2011). The everyday costs of poverty in childhood: a review of qualitative research exploring the lives and experiences of low-income children in the UK. Children and Society, 25, 73-84.

Sawyer, M. G., Streiner, D. L., \& Baghurst, P. (1998). The influence of distress on mothers' and fathers' reports of childhood emotional and behavioral problems. Journal of Abnormal Child Psychology, 26(6), 407-414.

Seeman, M. V. (2015). Schizophrenia and motherhood. In Reupert, A., Maybery, D., Nicholson, J., Gopfert, M., Seeman, M. V. (Eds), Parental psychiatric disorder: distressed parents and their families, (3rd Ed.) (pp. 107-116). Cambridge: Cambridge University Press.

Siegenthalar, E., Munder, T., \& Egger, M. (2012). Effect of preventive interventions in mentally ill parents on the mental health of the offspring: systematic review and meta-analysis. Journal of the American Academy of Child and Adolescent Psychiatry, 51(1), 8-17.

Slade, T., Johnston, A., Oakley Browne, M. A., Andrews, G., \& Whiteford, H. (2009). 2007 National Survey of Mental Health and Wellbeing: methods and key findings. Australian and New Zealand Journal of Psychiatry, 43, 594-605.

Stallard, P., Norman, P., Huline-Dickens, S., Salter, E., \& Cribb, J. (2004). The effects of parental mental illness upon children: a descriptive study of the views of parents and children. Clinical Child Psychology and Psychiatry, 9(1), 39-52.

Straussner, S. L. A., \& Huff-Fewell, C. (2011). Children of parents who abuse alcohol and other drugs. In Reupert, A., Maybery, D., Nicholson, J., Gopfert, M., Seeman, M. V. (Eds), Parental psychiatric disorder: distressed parents and their families, (3rd Ed.) (pp. 138-153). Cambridge: Cambridge University Press. 
Suarez, E. B., Lafreniere, G., Harrison, J. (2016). Scoping review of interventions supporting mothers with mental illness: key outcomes and challenges. Community Mental Health Journal, 52, 927-936.

The Royal Australian and New Zealand College of Psychiatrists. (2016). Position Statement 56. Children of parents with mental illness. Faculty of Child and Adolescent Psychiatry.

van Santvoort, F., Hosman, C. M. H., Janssens, J. M. A. M., van Doesum, K. T. M., Reupert, A., \& van Loon, L. M. A. (2015). The impact of various parental mental disorders on children's diagnoses: a systematic review. Clinical Child and Family Psychology Review, 18, 281-299.

Weissman, M. M. (2016). Children of depressed parents - a public health opportunity. JAMA Psychiatry, 73(3), 197-198.

Weissman, M. M., Wickramaratne, P., Nomura, Y., Waner, V., Pilowsky, D., \& Verdeli, H. (2006). Offspring of depressed parents: 20 years later. American Journal of Psychiatry, 163, 1001-1008.

Yoshikawa, H., Aber, J. L., \& Beardslee, W. R. (2012). The effects of poverty on the mental, emotional, and behavioural health of children and youth. American Psychologist, 67(4), 272-284.

Zubrick, S. R., Lawrence, D., Johnson, S., \& Hafekost, J. (2015). Measuring severity of mental disorders with the Young Minds Matter parent/carer-reported impact items. Technical Report. Perth: Telethon Kids Institute, The University of Western Australia. 
Table 1 - Proportion of primary, secondary or either carer ever having mental health problems, distress levels and interference of problems with daily activities

\begin{tabular}{|c|c|c|c|c|c|}
\hline $\begin{array}{l}\text { Category of mental health } \\
\text { problems }\end{array}$ & $\mathbf{n}$ & $\%$ & $95 \% \mathrm{CI}$ & $\begin{array}{r}\text { High-very high } \\
\text { psychological } \\
\text { distress }^{b}\end{array}$ & $\begin{array}{r}\% \text { with } \\
\text { interference } \\
\text { most or all of } \\
\text { the time }\end{array}$ \\
\hline Primary carer & \multicolumn{5}{|c|}{ All families $(N=6,310)$} \\
\hline Bipolar or psychotic disorder & 88 & 1.5 & $(1.2-1.8)$ & 44.2 & 30.2 \\
\hline Alcohol or drug dependence & 99 & 1.6 & $(1.2-1.9)$ & 27.8 & 20.8 \\
\hline Anxiety and depression & 664 & 10.6 & $(9.7-11.4)$ & 34.6 & 12.1 \\
\hline Depression only & 803 & 13.1 & $(12.1-14.1)$ & 14.7 & 7.6 \\
\hline Anxiety disorders only & 533 & 8.2 & $(7.5-8.9)$ & 12.8 & 3.8 \\
\hline Other mental health problem & 137 & 2.3 & $(1.8-2.7)$ & 32.4 & 23.5 \\
\hline Any mental health problem & 2,324 & 37.2 & $(35.7-38.7)$ & 22.7 & 10.5 \\
\hline
\end{tabular}

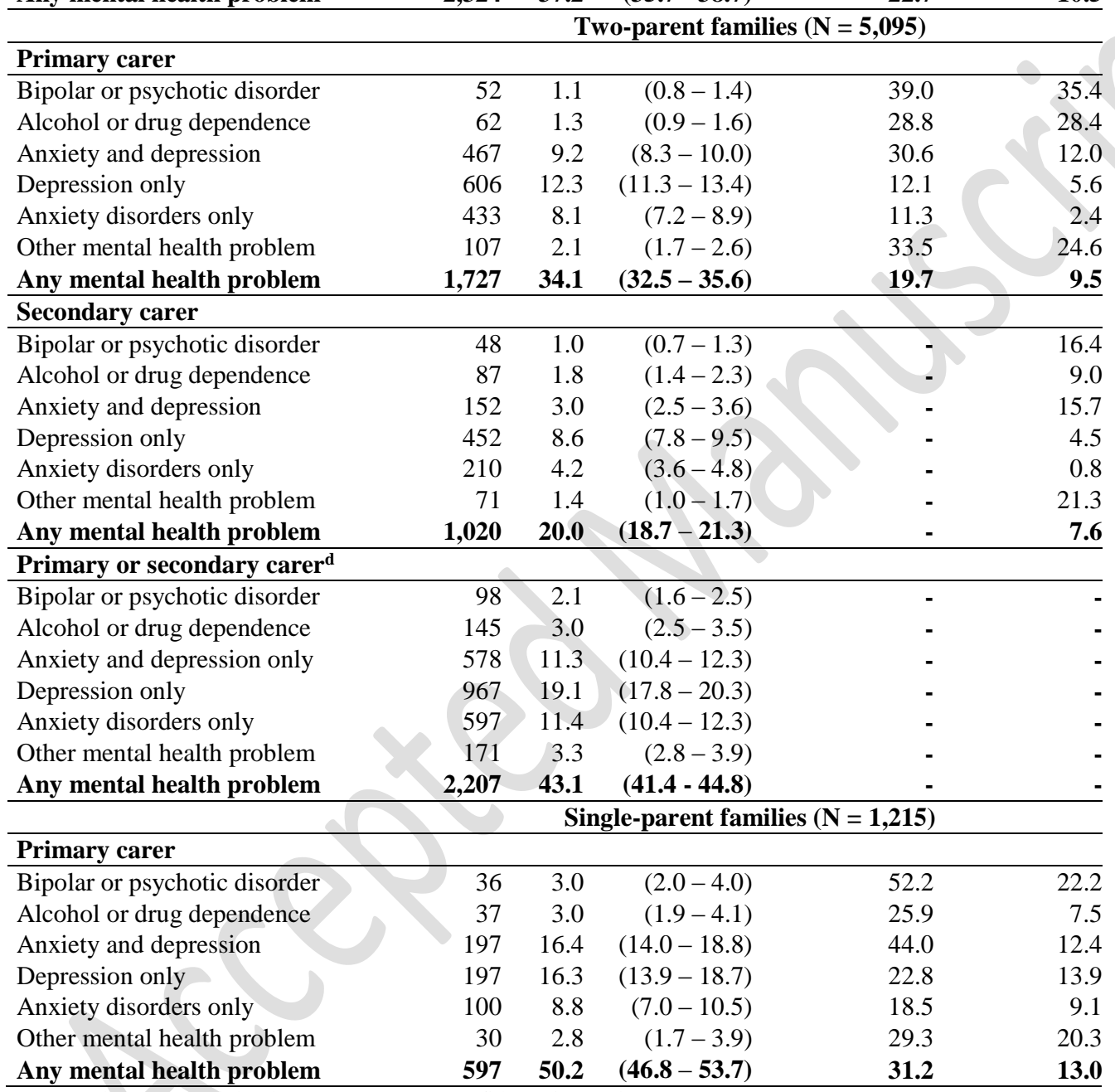

$\mathrm{n}=$ sample $\mathrm{n} ; \%=$ weighted percentage; $\mathrm{CI}=$ Confidence intervals

${ }^{a}$ Primary carer reports that they have ever been told by a doctor or health professional that they had a mental health problem. Anxiety disorders include panic attacks, PTSD, OCD and any other anxiety disorder. Bipolar or psychotic disorder, and alcohol or drug dependence may also include anxiety and/or depression. Other mental health problem includes ADHD and other unspecified problems, and may also include anxiety and/or depression.

${ }^{b}$ Psychological distress in past 4 weeks measured by Kessler Psychological Distress Scale (K10) - 12 missing cases. 'Indicates how much mental health problems have interfered with the primary carer's daily activities since the study child was born -11 cases with missing data.

${ }^{\mathrm{d}}$ This can be interpreted as either the primary or secondary carer having a diagnoses in the respective categories but doesn't preclude the other carer having a diagnosis in another category. 
Table 2 - Proportion of all families with specific socioeconomic characteristics by category of primary carer mental health problem $(\mathrm{N}=6,310)$

\begin{tabular}{|c|c|c|c|c|c|c|c|c|c|c|}
\hline \multirow[t]{2}{*}{ Category of problem ${ }^{a}$} & \multicolumn{2}{|c|}{ Below poverty line ${ }^{b}$} & \multicolumn{2}{|c|}{ IRSED Quintile ${ }^{\mathrm{c}}$} & \multicolumn{2}{|c|}{$\begin{array}{l}\text { No employed } \\
\text { parent }^{\mathrm{d}}\end{array}$} & \multicolumn{2}{|c|}{$\begin{array}{l}2 \text { or more indicators } \\
\text { of financial strain }\end{array}$} & \multicolumn{2}{|c|}{ Sole parent } \\
\hline & $\%$ & $95 \% \mathrm{CI}$ & $\%$ & $95 \% \mathrm{CI}$ & $\%$ & $95 \% \mathrm{CI}$ & $\%$ & $95 \% \mathrm{CI}$ & $\%$ & $95 \% \mathrm{CI}$ \\
\hline Bipolar or psychotic disorder & 42.2 & $(30.9-53.5)$ & 37.0 & $(25.1-48.9)$ & 37.1 & $(26.3-47.8)$ & 38.2 & $(27.2-49.3)$ & 41.8 & $(30.9-52.6)$ \\
\hline Alcohol or drug dependence & 36.8 & $(26.0-47.6)$ & 32.7 & $(20.9-44.4)$ & 32.7 & $(22.8-42.6)$ & 37.6 & $(26.8-48.3)$ & 43.6 & $(33.1-54.1)$ \\
\hline Anxiety and depression & 30.2 & $(26.3-34.0)$ & 19.4 & $(14.4-24.4)$ & 17.6 & $(14.0-21.2)$ & 20.2 & $(16.7-23.6)$ & 31.1 & $(27.0-35.2)$ \\
\hline Depression only & 25.7 & $(21.9-29.5)$ & 20.8 & $(15.9-25.8)$ & 13.4 & $(10.6-16.3)$ & 13.2 & $(10.5-15.8)$ & 25.2 & $(21.5-28.8)$ \\
\hline Anxiety disorders only & 20.8 & $(16.8-24.9)$ & 13.5 & $(9.0-18.0)$ & 14.0 & $(10.7-17.3)$ & 11.1 & $(8.1-14.1)$ & 22.3 & $(18.1-26.5)$ \\
\hline Other mental health problem & 31.3 & $(22.3-40.2)$ & 31.3 & $(20.8-41.8)$ & 16.8 & $(9.4-24.2)$ & 19.3 & $(10.9-27.7)$ & 25.8 & $(17.4-34.2)$ \\
\hline Any mental health problem & 27.4 & $(24.9$ - 29.9) & 20.6 & $(16.3-24.9)$ & 16.7 & $(14.5-18.9)$ & 17.1 & $(15.3-19.0)$ & 27.7 & $(25.3-30.1)$ \\
\hline $\begin{array}{l}\text { Primary carer has no mental } \\
\text { health problem }\end{array}$ & 17.8 & $(15.9-19.6)$ & 15.9 & $(12.6-19.3)$ & 9.1 & $(7.8-10.4)$ & 7.0 & $(6.0-7.9)$ & 15.9 & (14.4-17.5) \\
\hline
\end{tabular}

= weighted percentage; $\mathrm{CI}=$ Confidence Intervals

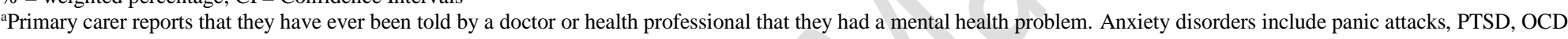
and any other anxiety disorder. Bipolar or psychotic disorder, and alcohol or drug dependence may also include anxiety and/or depression. Other mental health problem includes ADHD and other unspecified problems, and may also include anxiety and/or depression.

${ }^{b}$ Below Poverty Line is calculated as those families at 50\% of the median equivalised annual gross household income (21.3\% of all families)

'The lowest or most disadvantaged quintile on the Australian Bureau of Statistics Index of Relative Socio-economic Disadvantage (IRSED).

${ }^{\mathrm{d}}$ Either both parents unemployed in a two-parent family or sole parent unemployed.

${ }^{\text {e}}$ Indicators of financial strain in past 12 months were either not being able to pay utility bills on time, not being able to pay mortgage or rent on time, going without meals or having sought assistance from a welfare or community organisation. 
Table 3 - Prevalence of child mental disorders by primary or secondary carer mental health problems and family structure

\begin{tabular}{|c|c|c|c|c|c|c|c|c|}
\hline \multirow{3}{*}{ Category of problems ${ }^{a}$} & \multirow{2}{*}{$\begin{array}{l}\text { Sole-parent family } \\
\qquad(\mathrm{N}=\mathbf{1 , 2 1 5}) \\
\text { PC only }\end{array}$} & & & \multicolumn{2}{|l|}{$\begin{array}{l}\text { Two-parent family } \\
\qquad(\mathrm{N}=\mathbf{5 , 0 9 5})\end{array}$} & \multicolumn{3}{|c|}{$\begin{array}{l}\text { All families } \\
(N=6,310)\end{array}$} \\
\hline & & \multicolumn{2}{|c|}{ PC only ${ }^{b}$} & SC only ${ }^{b}$ & Both PC and SC & \multicolumn{3}{|c|}{ PC } \\
\hline & $95 \% \mathrm{CI}$ & $\%$ & $95 \% \mathrm{CI}$ & $95 \% \mathrm{CI}$ & $95 \% \mathrm{CI}$ & $95 \% \mathrm{CI}$ & $\mathbf{R R}$ & $95 \% \mathrm{CI}$ \\
\hline \multicolumn{9}{|c|}{ Child/Adolescent Any Mental Disorder } \\
\hline Bipolar or psychotic disorder & $51.6(33.7-69.5)$ & 24.0 & $(11.7-36.2)$ & $22.1 \quad(6.8-37.4)$ & & $34.4(24.1-44.6)$ & 3.9 & $(2.8-5.4)$ \\
\hline Alcohol or drug dependence & $25.0 \quad(9.7-40.2)$ & 32.9 & $(19.7-46.1)$ & $29.2(18.3-40.1)$ & np & $32.1(21.9-42.3)$ & 3.6 & $(2.6-5.1)$ \\
\hline Anxiety and depression & $33.7(26.3-41.2)$ & 22.9 & $(18.4-27.4)$ & $17.1 \quad(8.9-25.2)$ & $(7.0-31.3)$ & $26.0(22.1-29.8)$ & 3.0 & $(2.4-3.6)$ \\
\hline Depression only & $27.4 \quad(20.9-33.9)$ & 18.0 & $(14.3-21.6)$ & $15.2(11.0-19.3)$ & $(5.1-17.9)$ & $19.5(16.6-22.4)$ & 2.2 & $(1.8-2.7)$ \\
\hline Anxiety disorders only & $22.7 \quad(14.4-30.9)$ & 14.2 & $(10.3-18.0)$ & $9.8 \quad(5.0-14.6)$ & $(6.7-27.5)$ & $16.2(12.7-19.7)$ & 1.8 & $(1.4-2.4)$ \\
\hline Other mental health problem & $29.2(11.4-46.9)$ & 38.8 & $(26.9-50.7)$ & $26.2(14.8-37.5)$ & np & $34.6(24.8-44.3)$ & 3.9 & $(2.9-5.3)$ \\
\hline Any mental health problem & $30.0 \quad(25.6-34.4)$ & 19.1 & $(16.7-21.5)$ & $(7.4-13.7)$ & $(17.9-25.9)$ & $22.6(20.6-24.7)$ & 2.6 & $(2.2-3.0)$ \\
\hline No mental health problem & $14.6(11.4-17.8)$ & 7.3 & $(6.2-8.3)$ & $7.3 \quad(6.2-8.3)$ & $(6.2-8.3)$ & $(7.7-9.8)$ & - & - \\
\hline
\end{tabular}

Child and adolescent disorders are identified by the DISC-IV (either an anxiety disorder, major depressive disorder, ADHD or conduct disorder).

$\mathrm{np}=$ not available for publication because of small cell sizes, but included in totals where applicable. PC = primary carer; SC = secondary care

a Primary carer reports that they have ever been told by a doctor or health professional that they had a mental health problem. Anxiety disorders include panic attacks, PTSD, OCD

and any other anxiety disorder. Bipolar or psychotic disorder, and alcohol or drug dependence may also include anxiety and/or depression. Other mental health problem includes

$\mathrm{ADHD}$ and other unspecified problems, and may also include anxiety and/or depression.

${ }^{\mathrm{b}}$ The figures for 'PC only' and 'SC only' in two-parent families can be interpreted as just one of the carers having a diagnoses in that category of problem but does not preclude the other carer having a

diagnosis in another category. 
Table 4 - Prevalence of child anxiety disorders, major depressive disorder and externalizing disorders (ADHD or conduct disorder) by primary or secondary carer mental health problems and family structure.

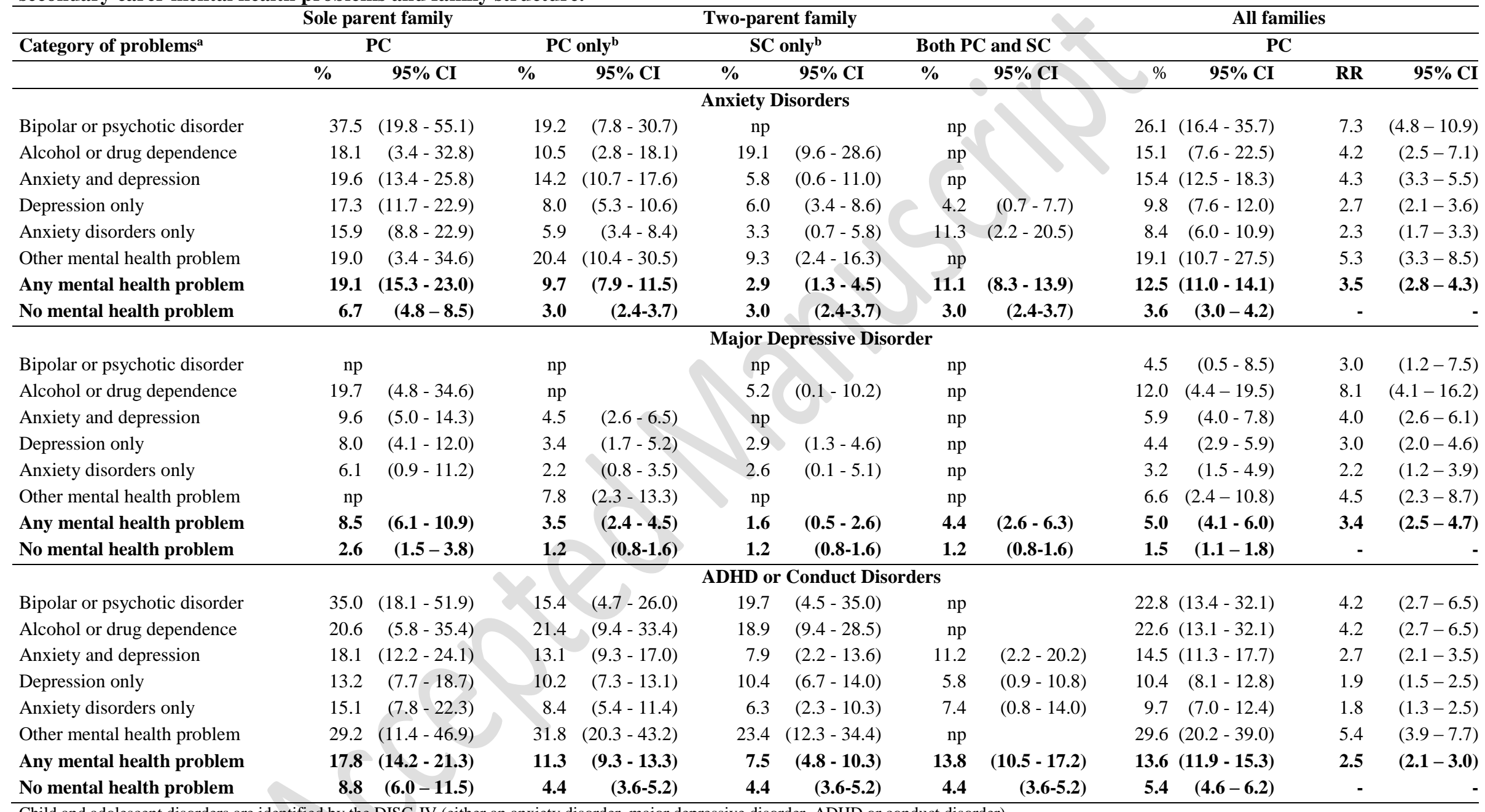

Child and adolescent disorders are identified by the DISC-IV (either an anxiety disorder, major depressive disorder, ADHD or conduct disorder).

$\mathrm{np}=$ not available for publication because of small cell sizes, but included in totals where applicable. $\mathrm{PC}=$ primary carer; $\mathrm{SC}=$ secondary carer.

$\%=$ weighted proportion; $\mathrm{CI}=$ Confidence intervals; $\mathrm{RR}=$ relative risk of the child having any disorder if the PC has that category of $\mathrm{MH}$ problem, compared to if the $\mathrm{PC}$ has no $\mathrm{MH}$ problem.

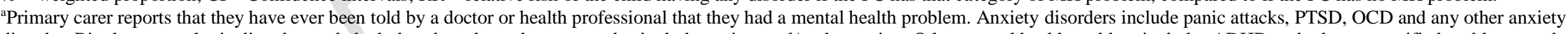
disorder. Bipolar or psychotic disorder, and alcohol or drug dependence may also include anxiety and/or depression. Other mental health problem includes ADHD and other unspecified problems, and may also include anxiety and/or depression. 
${ }^{\mathrm{b}}$ The figures for 'PC only' and 'SC only' in two-parent families can be interpreted as just one of the carers having a diagnoses in that category of problem but does not preclude the other carer having a diagnosis in another category.

Table 5 - Prevalence and severity of any child/adolescent mental disorder by primary carer diagnosis and impact on functioning in all families $(\mathrm{N}=$ 6,310)

\begin{tabular}{|c|c|c|c|c|c|c|c|c|c|c|c|}
\hline \multirow[b]{3}{*}{$\begin{array}{l}\text { Primary carer MH diagnosis }{ }^{\text {a }} \text { /interference } \\
\text { with daily activities }\end{array}$} & \multirow[b]{3}{*}{$\mathbf{n}$} & \multicolumn{10}{|c|}{ Severity of child mental disorder } \\
\hline & & & & \multicolumn{2}{|c|}{ Mild } & \multicolumn{2}{|c|}{ Moderate } & \multicolumn{2}{|c|}{ Severe } & \multicolumn{2}{|c|}{ Total } \\
\hline & & $\%$ & $95 \% \mathrm{CI}$ & $\%$ & $95 \% \mathrm{CI}$ & $\%$ & $95 \% \mathrm{CI}$ & $\%$ & $95 \% \mathrm{CI}$ & $\%$ & $95 \% \mathrm{CI}$ \\
\hline No MH diagnosis/none or a little of the time & 3756 & 59.3 & $(57.8-60.8)$ & 5.3 & $(4.5-6.1)$ & 1.4 & $(1.0-1.8)$ & 0.7 & $(0.4-0.9)$ & 7.4 & $(6.4-8.3)$ \\
\hline No $\mathrm{MH}$ diagnosis/some, most or all of the time & 221 & 3.4 & $(2.9-4.0)$ & 12.6 & $(7.2-17.9)$ & 13.8 & $(8.6-19.1)$ & 6.1 & $(3.0-9.2)$ & 32.5 & $(25.6-39.3)$ \\
\hline MH diagnosis/none or a little of the time & 1356 & 21.6 & $(20.6-22.7)$ & 10.9 & $(9.1-12.7)$ & 4.0 & $(2.9-5.1)$ & 1.3 & $(0.7-1.9)$ & 16.2 & $(14.0-18.4)$ \\
\hline MH diagnosis/some of the time & 734 & 11.7 & $(10.8-12.6)$ & 14.1 & $(11.3-16.8)$ & 7.2 & $(5.0-9.3)$ & 5.5 & $(3.6-7.4)$ & 26.8 & $(23.1-30.3)$ \\
\hline MH diagnosis/most or all of the time & 232 & 3.9 & $(3.4-4.4)$ & 19.1 & $(13.4-24.9)$ & 13.9 & $(9.3-18.4)$ & 13.1 & $(8.1-18.1)$ & 46.1 & $(38.9-53.3)$ \\
\hline
\end{tabular}

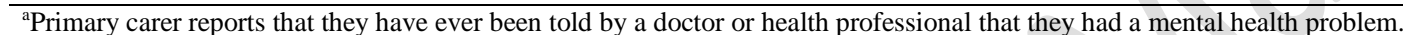

${ }^{b}$ Indicates how much mental health problems have interfered with the primary carer's daily activities since the study child was born - 11 cases with missing data. 